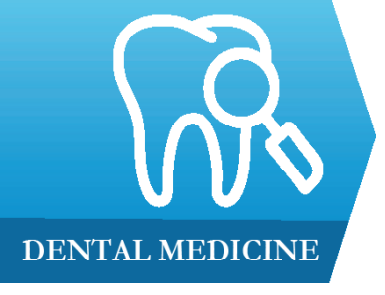

Department of Conservative Dentistry and Endodontics, College of Dental Science \& Hospital, Rau, Indore Madhya Pradesh Medical Science University, India
DOI: $10.15386 / \mathrm{mpr}-1488$

Manuscript received: 13.10.2019

Received in revised form: 22.02 .2020

Accepted: 08.03.2020

Address for correspondence:

manasi.kewlani@gmail.com

This work is licensed under a Creative Commons Attribution-NonCommercialNoDerivatives 4.0 International License

\section{Comparative evaluation of the effect of decontamination protocol on the shear bond strength of eighth generation bonding agent to contaminated dentin: an in vitro study}

Manasi Kewlani, Suparna Ganguly Saha, Anuj Bhardwaj, Mainak Kanti Saha, Prashansa Vijaywargiya, Shikha Jain, S. V. Sai Prasad

\begin{abstract}
Introduction. This in vitro study aims to evaluate and compare the effect of decontamination protocol - based on sodium hypochlorite - $\mathrm{NaOCl}$, as a decontaminating agent, followed by the application of a collagen cross-linking agent like sodium ascorbate or Proanthocyanidin on the shear bond strength of eighth generation bonding agent to contaminated dentin (saliva, blood and blood followed by hemostatic agent).

Methods. The buccal surfaces of extracted premolars were flattened to expose the dentine. The teeth were then randomly divided into 3 groups based on the contaminant used (saliva, blood and blood followed by hemostatic agent). Decontamination protocol was carried out with sodium hypochlorite followed by application of either of the two collagen cross-linking agents (sodium ascorbate/proanthocyanidin) in each group. FuturaBond DC (eighth generation) bonding agent was used to bond composite to dentin surfaces which was subjected to shear bond strength testing. Data was analyzed by one-way ANOVA and Tukey test.

Results. All the groups (contaminated with saliva, blood, or hemostatic agent) showed a decrease in shear bond strength of composite resin to dentin. The shear bond strength of composite resin to dentin was least affected by contamination with saliva followed by contamination with blood and was most affected by contamination with a hemostatic agent.

The decontamination protocol carried out showed that the combination of $\mathrm{NaOCl}$ and Proanthocyanidin improved the shear bond strength of composite to dentin significantly more than the combination of $\mathrm{NaOCl}$ and sodium ascorbate or of sodium hypochlorite used alone.
\end{abstract}

Keywords: contaminants, dentin bonding agents, shear bond strength

\section{Background and aims}

Isolation is a pre-requisite for the success of bonded restorations which are highly technique-sensitive and extremely vulnerable to contamination. Saliva, blood, excess moisture, hemostatic agents, gingival crevicular fluid, handpiece oil etc. are common contaminants which exert adverse effects on adhesive systems [1].

Contamination by blood and saliva negatively affects the bonding between composite restorations and dentin, thereby leading to undesirable consequences like micro-leakage, secondary caries and marginal staining which ultimately lead to failure of the restoration [2].

Aluminum chloride (20 to $25 \%$ ) which is a common hemostatic agent interferes with the adhesion of bonded restorations [3]. 
Moreover, dentin is a complex non-homogeneous substrate with high organic and moisture content, making bonding a further challenge $[1,4]$

Sodium hypochlorite, used as part of the decontamination protocol after contact of the dentin with saliva, blood or hemostatic agent, possesses hemolytic and hemoglobinolytic action. In addition to serving as a solvent for the organic debris, when applied on contaminated acid etched dentin, it also acts as a deproteinizing agent, thus modifying the surface of dentinal substrate [5].

Literature has shown that the bond strength of composite resin to dentin may be enhanced by additional treatment with an antioxidant and collagen cross linking agent such as sodium ascorbate [6].

Naturally occurring antioxidants such as grape seeds containing oligomeric proanthocyanidin complexes (OPC) have free radical scavenging activity as well as antibacterial, antiviral, anti-carcinogenic, antiinflammatory, anti-allergic and vasodilatory actions. They also serve as collagen cross linking agents, thereby improving the bond strength of adhesive systems to dentin [6].

Attempts to improve the bond strength, as well as to simplify the bonding technique, have now led to the introduction of the eighth generation dentin adhesive systems. This generation of self-etch adhesive systems which incorporate nanotechnology, claim to have superior bond strength to enamel as well as to dentin, in addition to better stress absorption properties and longer shelf life.

Thus, the aim of this study was to evaluate and compare the effect of decontamination protocol on the shear bond strength of eighth generation bonding agent to dentin contaminated by saliva, blood and blood followed by hemostatic agent using $10 \%$ sodium hypochlorite, followed by application of a collagen crosslinking agents like $10 \%$ sodium ascorbate (Na Ascorbate) or 5\% Proanthocyanidin (PA).

\section{Methods}

Sample size calculation using $G^{*}$ power software revealed that minimum of 5 samples per group would be required to detect a difference in mean value of shear bond strength between 13 groups at an alpha of 0.05 and power of $80 \%$.

80 intact premolar teeth freshly extracted for orthodontic and periodontal reasons were collected and stored according to CDC (Centers for Disease Control and Prevention) guidelines for infection control.

Buccal surfaces of premolars were sliced with water cooled diamond disc to remove enamel and expose 5-6 $\mathrm{mm}$ area of dentin. The teeth were then mounted on self-cure acrylic resin mold.
The samples were stored in distilled water until used for bonding. Prior to bonding, fresh whole blood and saliva was collected from the chief investigator in a sterile beaker/test-tube and were used immediately.

All the teeth were randomly divided into 4 groups A, B, C and D, 20 in each group, labeled according to the contaminant used (Figure 1).

\section{- Control Group D}

No contamination was done. Bonding agent (FuturaBond DC, Voco) was applied according to manufacturer's instructions and light curing was done for 40 seconds.

The three groups A, B and C were further divided into 4 sub-groups of 5 teeth each.

$$
\begin{aligned}
& A \rightarrow A_{1}, A_{2}, A_{3}, A_{4} \text { (contaminated with saliva) } \\
& B \rightarrow B_{1}, B_{2}, B_{3}, B_{4} \text { (contaminated with blood) } \\
& C \rightarrow C_{1}, C_{2}, C_{3}, C_{4} \text { (contaminated with hemostatic }
\end{aligned}
$$
agent containing aluminium chloride)

Contamination was done for 15 seconds.

- Sub-groups $A_{1}, B_{1}$ and $C_{1}$ :

No decontamination was done. After rinsing the dentin surface, bonding agent was applied according to manufacturer's instructions and light curing was done for 40 seconds.

- Sub-groups $\mathrm{A}_{2}, \mathrm{~B}_{2}$ and $\mathrm{C}_{2}(10 \% \mathrm{NaOCl})$ :

Contaminated dentin surface was rinsed with $10 \% \mathrm{NaOCl}$ (Loba Chemie Pvt. Ltd. Mumbai, India) for 60 seconds and then washed with water using air-water syringe for another 60 seconds.

- Sub-groups $\mathrm{A}_{3}, \mathrm{~B}_{3}$ and $\mathrm{C}_{3}(10 \% \mathrm{NaOCl}+10 \%$ Sodium Ascorbate):

Contaminated dentin surface was rinsed with $10 \%$ $\mathrm{NaOCl}$ for 60 seconds followed by application of $10 \%$ $\mathrm{Na}$ Ascorbate (Qualikems Fine Chem Pvt. Ltd, Vadodara, India) for 60 seconds and then washed with water using air-water syringe for another 60 seconds.

- Sub-groups $\mathrm{A}_{4}, \mathrm{~B}_{4}$ and $\mathrm{C}_{4}(10 \% \mathrm{NaOCl}+5 \%$

\section{Proanthocyanidin):}

Contaminated dentin surface was rinsed with $10 \% \mathrm{NaOCl}$ for 60 seconds followed by application of 5\% Proanthocyanidin (Medlife International Pvt. Ltd, Bengaluru, India) for 10 minutes and then washed with water for another 60 seconds. After rinsing, excess water was removed with cotton pellet and bonding agent (FuturaBond DC, Voco) was applied with the help of the applicator tip followed by light curing for 20 seconds.

Teflon tube $(3 \mathrm{~mm} \times 3 \mathrm{~mm})$ was applied on the sliced dentinal surface. The Teflon tube was then filled with composite resin (FilTek Z350 3M ESPE) in two increments (1.5 mm each) and each increment was compressed firmly onto the exposed dentin surface and light cured for 40 seconds. The Teflon tube was then removed and additional curing of resin cylinder was done. 


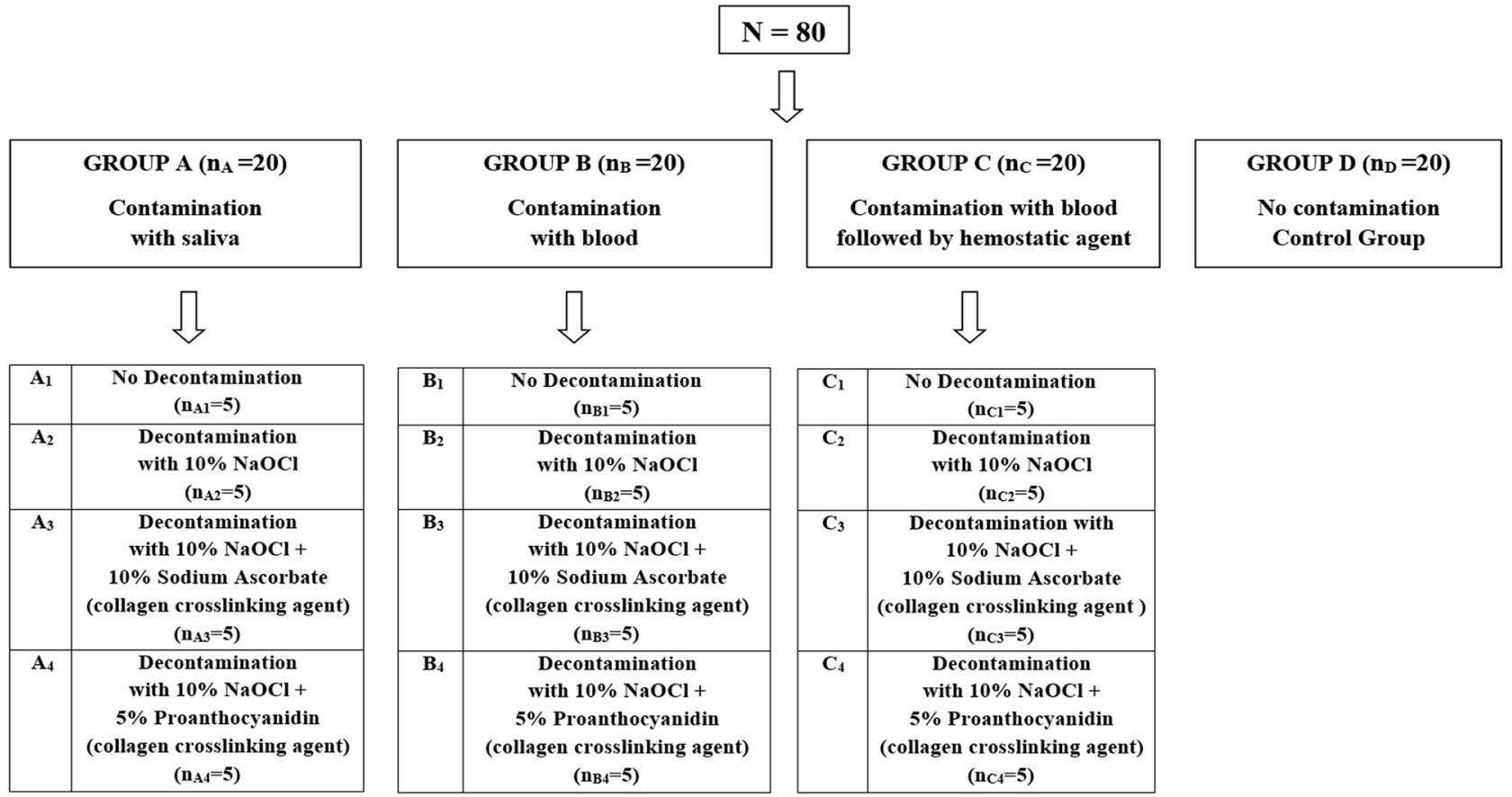

Figure 1. Schematic representation of procedure done in the study.

The prepared specimens were stored in distilled water at $37^{\circ} \mathrm{C}$ for 24 hours and then mounted on universal testing machine for shear bond strength testing.

A knife edge chisel shaped shearing rod was used for de-bonding of composite resin cylinders at a crosshead speed of $0.5 \mathrm{~mm} /$ minute. The maximum load in MPa required to de-bond the composite resin cylinders was calculated.

\section{Statistical analysis}

Mean and standard deviation were estimated for each study group. Normality of the data was tested in each group by using Kolmogorov-Smirnov test. Mean values were compared between different study groups by using One way ANOVA followed by Post-hoc Tukey procedure.

\section{Results}

The maximum load in Newtons required to de-bond the composite resin cylinders was recorded to calculate the stress at fracture, i.e. the shear bond strength in MPa. Data were summarized as mean \pm SD. The following results were obtained (Table I).

Effect of contamination on shear bond strength to dentin

saliva $>$ blood $>$ hemostatic agent

Effect of decontamination protocol on shear bond strength of composite to dentin

$10 \% \mathrm{NaOCl}+5 \% \mathrm{PA}>10 \% \mathrm{NaOCl}+10 \%$ Sodium Ascorbate $>10 \% \mathrm{NaOCl}$

Table I. Intergroup comparison of mean shear bond strength values (MPa) of eighth generation dentin bonding agent to dentin contaminated by saliva, blood, and blood followed by hemostatic agent.

\begin{tabular}{ll|c|c|c|c|c|c} 
Group & Mean & Minimum & Maximum & \pm SD & 'F' value & ' $\mathbf{p}$ ' value \\
Group A Cont. with saliva & 24.0 & 19.4 & 31.4 & 3.31 & & \\
Group B Cont. with blood & 21.4 & 17.2 & 26.2 & 2.82 & 12.61 & 0.000 Highly significant \\
Group C Cont. with hemostatic agent & 19.8 & 16.4 & 23.8 & 2.06 & 2.44 &
\end{tabular}




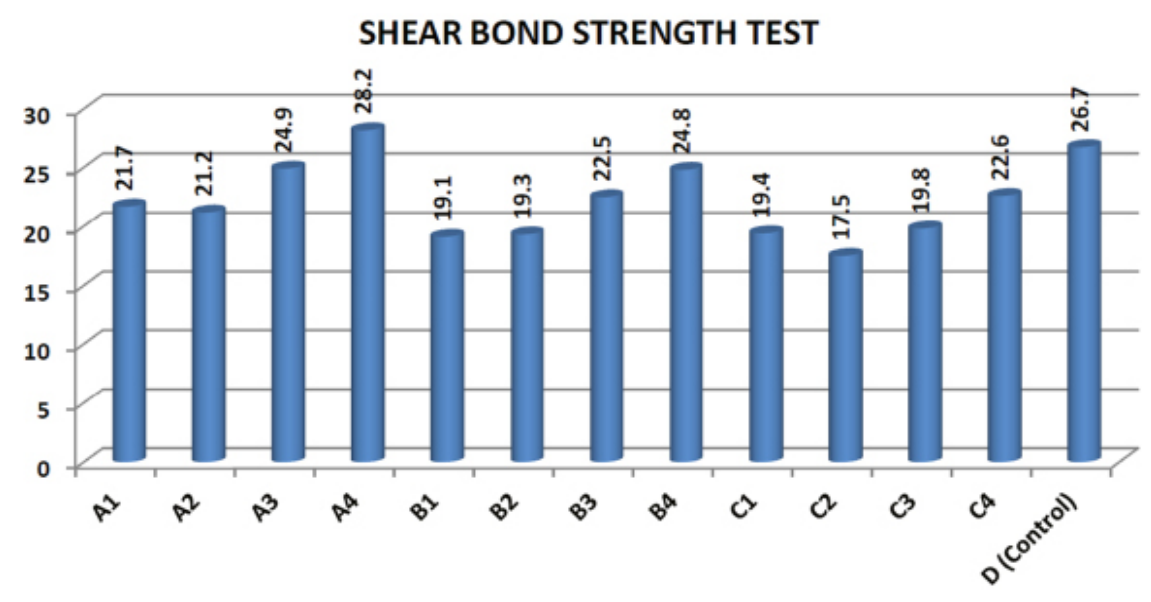

Mean

Figure 2. Intergroup sub-group comparison of mean shear bond strength values (MPa) of eighth generation dentin bonding agent to dentin based on decontamination protocol used after contamination with saliva, blood, and blood followed by hemostatic agent.

\section{Discussion}

The increasing demand for esthetic restorations has resulted in a drastic increase in their clinical applications leading to intensive research on adhesives. The fundamental requirement of bonded restorations is a durable adhesion of the composite resin to the tooth structure [7].

In order to generate an adequate seal between the restoration and tooth surface, successful adhesion without contamination is necessary. However, dental adhesives and composites are highly vulnerable to contaminants like saliva, blood, or hemostatic agent [1].

The results of the present study showed that all the contaminants (Saliva, Blood and Hemostatic agent) had a negative impact on the bond strength to dentin (Table I).

The comparison between the three contaminants revealed that blood affected the bond strength more deleteriously when compared to saliva contamination. The reason for this could be attributed to the greater mechanical barrier created by blood in comparison to saliva owing to the difference in the type and amount of organic andinorganic elements in the blood [7].

Maximum negative impact was seen when blood contaminated samples were treated with hemostatic agent containing aluminium chloride. The calcium ions present in the hydroxyapatite crystals of enamel and dentin are replaced by aluminium ions present in the hemostatic agent which inhibits the effective demineralization of these tissues. Decrease in bond strength may be attributed to deposition of aluminium in the form of unbound minerals on the dentin surface forming a residual layer leading to decreased adhesive infiltration into dentin thereby impeding the bonding procedures [8].
To circumvent this problem of contamination, a decontamination protocol was carried out with different agents and their effect on the bond strength was evaluated and compared.

Decontamination by rinsing with water or re-etching has not proved to be an effective method for regaining adequate bond strength [5].

Therefore, various effective decontaminants have been used in the present study including $\mathrm{NaOCl}$, Sodium Ascorbate and PA.

$\mathrm{NaOCl}$ is a commonly used endodontic irrigant and decontaminant. It has antimicrobial and tissue-dissolving properties. $\mathrm{NaOCl}$ being a strong oxidizing agent leaves behind an oxygen rich layer on the dentin surface improving the bond strength of resin composites [6].

The relatively lower bond strength obtained with $\mathrm{NaOCl}$ alone may be attributed to the production of oxygen due to the disintegration of $\mathrm{NaOCl}$ to $\mathrm{NaCl}$ and oxygen. The oxygen ions released in this process occlude the dentinal tubules, preventing polymerization of the adhesive agents [5].

$\mathrm{NaOCl} \rightarrow \mathrm{NaCl}+[\mathrm{O}]$

To enhance bond strength further, application of antioxidant and collagen cross-linking agent like sodium ascorbate has been shown to significantly increase bond strength in $\mathrm{NaOCl}$ treated dentin [6].

Sodium ascorbate is reported to reverse the negative effects of $\mathrm{NaOCl}$ on polymerization of dentin bonding agent. $\mathrm{Na}$ ascorbate restores the bond strength by acting as an electron donor and scavenging free radicals, allowing complete polymerization without pre-mature termination of process [6]. 
$\mathrm{Na}$ ascorbate also eliminates vertical shag-carpetlike nanoleakage pattern created by $\mathrm{NaOCl}$ due to incomplete penetration of resin into demineralized dentin [5].

Sodium ascorbate when used following the use of $\mathrm{NaOCl}$ was found to promote the polymerization reaction of free radicals of the adhesive agent without premature termination and reverses the disrupted bonding observed with $\mathrm{NaOCl}$ treated dentin.

In order to achieve the desired bond strength of composite resin to contamination free surface, proanthocyanidin has been used in this study, as it overcomes the shortcomings of other decontaminants.

Proanthocyanidin, a natural dentin biomodifier in adhesive dentistry is a plant flavonoid obtained from natural sources such as grape seed extract, cocoa beans, pine bark extract, cranberries, lemon tree bark, and hazel nut tree leaves. Grape seed extract was chosen for this study since it yields a $10 \%$ higher concentration of PAs [9].

PAs have been proven to be a safe antioxidant and collagen cross-linking agent in various clinical applications and dietary supplements. They have a significant role in enhancing the durability of resin-dentin bond strength. OPC (oligomeric proanthocyanidin complexes) contains multiple electron donor sites (hydroxyl sites) that allow it to bind to unstable molecules called free radicals by donating its hydrogen atoms. Hence, it has been postulated that PA hydrogen bond formation in multiple sites on collagen molecule reduces possible cleavage sites. The presence of gallic acid also increases the free radical scavenging activity $[9,10]$.

PAs also functions as an effective proteolytic inhibitor and dentin collagen matrix stabilizer. Collagen in biological tissue is strengthened by the formation of native cross links which provides the fibrillar resistance against enzymatic degradation as well as greater nano-mechanical properties. PAs can increase the number of inter and intramolecular cross links [9].

Proanthocyanidin proves to be of utmost importance for the clinical longevity of bonded restorations.

According to this study, combination of $\mathrm{NaOCl}$ and Proanthocyanidin showed superior results in regaining the shear bond strength of composite resin to dentin when compared to a combination of $\mathrm{NaOCl}$ and $\mathrm{Na}$ ascorbate and $\mathrm{NaOCl}$ alone (Figure 2).

The maximum shear bond strength obtained with Proanthocyanidin can be attributed to the scavenging potential of one of its constituent i.e. gallic acid. Collagen crosslinking and stabilization property of PA can be an another important factor for the increased bond strength [6].

\section{Conclusion}

Within the limitations of this study it may be concluded that:
- Saliva, blood, and hemostatic agent have a negative impact on the shear bond strength of composite resin to dentin.

- Contamination with hemostatic agent and blood adversely affects the bond strength more than the saliva contamination.

- Decontamination protocol carried out in this study showed that a combination of $\mathrm{NaOCl}$ and Proanthocyanidin improved the shear bond strength of composite to dentin significantly more when compared to a combination of $\mathrm{NaOCl}$ and sodium ascorbate or to sodium hypochlorite used alone.

\section{References}

1. Munaga S, Chitumalla R, Kubigiri SK, Rawtiya M, Khan S, Sajjan P. Effect of saliva contamination on the shear bond strength of a new self-etch adhesive system to dentin. J Conserv Dent. 2014;17:31-34.

2. Taneja S, Kumari M, Bansal S. Effect of saliva and blood contamination on the shear bond strength of fifth-, seventh-, and eighth-generation bonding agents: An in vitro study. J Conserv Dent. 2017;20:157-160.

3. Ajami AA, Kahnomoii MA, Kimyai S, Oskoee SS, Pournaghi-Azar F, Bahari M, et al. Effect of three different contamination removal methods on bond strength of a self-etching adhesive to dentin contaminated with an aluminium chloride hemostatic agent. J Contemp Dent Pract. 2013;14:26-33.

4. Kulkarni AS, Kokate S, Hegde V. The effect of saliva contamination on shear bond strength of two universal bonding agents-an in vitro study. J Clin Diagn Res. 2018;12(4):ZC6-ZC10.

5. Juneja R, Duhan J, Tewari S, Sangwan P, Bhatnagar N. Effect of blood contamination and decontamination protocols on acetone-based ethanol-based total etch adhesive systems. J Esthet Restor Dent. 2014;26:403-416.

6. Manimaran VS, Srinivasulu S, Rajesh Ebenezar AV, Mahalaxmi S, Srinivasan N. Application of a proanthocyanidin agent to improve the bond strength of root dentin treated with sodium hypochlorite. J Conserv Dent. 2011;14:306-308.

7. Shaikh A, Hegde V, Shanmugasundaram S. Effect of blood contamination and decontamination procedures on the micro-tensile bond strength of a new self-etch adhesive: An in-vitro study. Int J Experimental Dent Sci. 2017;6(2):80-83.

8. Anil A, Sekhar A, Thomas MS, Ginjupalli K. Hemostatic agents on the shear bond strength of self-adhesive resin. J Clin Exp Dent. 2015;7:e356-e360.

9. Nagpal R, Singh P, Singh S, Tyagi SP. Proanthocyanidin: A natural dentin biomodifier in adhesive dentistry. J Res Dent. 2016;4:1-6.

10. Liu Y, Wang Y. Proanthocyanidins' efficacy in stabilizing dentin collagen against enzymatic degradation: MALDITOF and FTIR analyses. J Dent. 2013;41:535-542 